\title{
PSICOLOGÍA Y VIH/SIDA EN MÉXICO: SU PREVENCIÓN CON BASE EN UN MODELO PSICOLÓGICO DE SALUD BIOLÓGICA
}

\author{
JULIO A. PIÑA * y SUSANA ROBLES* * \\ * Universidad de Sonora, México \\ ** Facultad de Estudios Superiores Iztacala, Universidad Nacional Autónoma de México
}

\begin{abstract}
En este trabajo se revisa brevemente el problema del VIH/SIDA en México y la manera en la que se le ha abordado desde una perspectiva institucional, es decir, desde una epidemiológica y social. Con base en un modelo psicológico de salud biológica se reseñan los resultados de diversas investigaciones realizadas en los tres últimos años, sugiriéndose una serie de criterios a tener en cuenta al momento de diseñar e instrumentar programas y estrategias dirigidas a la prevención de la infección por VIH.
\end{abstract}

Palabras clave: VIH/SIDA, modelo psicológico, prevención.

\section{Psychology and HIV/SIDA in Mexico: prevention from a psychological model of biologi- cal health}

In this paper, a brief review of the HIV/AIDS problem in Mexico and the way in which it has been boarded from an epidemiologic and social perspective it is presented here. Results of several researches made it with base in a psychological model of biological health are resumed, suggesting some criteria to derive programs and strategies directed to the prevention of the HIV infection.

Key words: HIV/AIDS, psychological model, prevention.

\section{INTRODUCCIÓN}

Más allá de lo que se esperaba una década atrás, con el correr de los años el del VIH/SIDA se ha convertido en un serio problema de salud pública para buena parte de los países del orbe, y México, por supuesto, no es la excepción. En nuestro país, de acuerdo con el último informe presentado por el Centro Nacional para la Prevención y Control del VIH/SIDA -CENSIDA-, desde 1983 hasta finales de $2002^{1}$ se habían notificado a las autoridades del sector salud 68 145 casos de SIDA (ver Cuadro 1). Empero, si se tienen en cuenta los fenómenos de retraso en la notificación (Anaya,

Correspondencia: Julio A. Piña, Guillermo Prieto 18, Col. Constitución, Hermosillo, Sonora (México), CP 83150. Correo-e: jpina@psicom.uson.mx. Susana Robles, Av. de los Barrios 1, Los Reyes Iztacala, Tlanepantla, Estado de México (México), CP 54090. Correoe: susana@campus.iztacala.unam.mx.

1 Datos actualizados hasta diciembre de 2003.
1997) y subrregistro de casos (Valdespino et al., 1995), que ascienden a 33 y $18,5 \%$, respectivamente, es posible que el número total de casos sea de aproximadamente 257420.

A lo anterior habría que añadir que en los años 2000 y 2001 el SIDA pasó a ocupar los lugares 16 y 17, respectivamente, como causa de mortalidad general en nuestro país, aunque en esos mismos años se ubicó en el lugar número siete como causa de mortalidad en personas en edad productiva, es decir, en personas cuyas edades oscilan entre los 17 y 44 años, lo que por sí sólo nos habla del innegable impacto que la citada patología está teniendo sobre este amplio sector de la población ${ }^{2}$.

2 Sobre todo si se analiza el problema en términos de la cuantificación de dos indicadores básicos: los años perdidos de vida potencial -APVP-y los años perdidos de vida productiva potencial - APVPP(cfr. Izazola, Valdéz, Sánchez y del Río, 1995; Magis y del Río, 2000). 
Cuadro 1: Casos de SIDA según las fechas de notificación y diagnóstico. Fuente: CENSIDA (2003)

\begin{tabular}{lcc}
\hline Año & $\begin{array}{c}\text { Casos notificados } \\
\text { por año }\end{array}$ & $\begin{array}{c}\text { Casos diagnosticados } \\
\text { por año }\end{array}$ \\
\hline 1983 & 6 & 60 \\
1984 & 6 & 198 \\
1985 & 29 & 349 \\
1986 & 246 & 673 \\
1987 & 518 & 1.485 \\
1988 & 905 & 2.069 \\
1989 & 1.605 & 2.260 \\
1990 & 2.587 & 3.516 \\
1991 & 3.155 & 3.625 \\
1992 & 3.210 & 3.985 \\
1993 & 5.058 & 3.942 \\
1994 & 4.111 & 3.968 \\
1995 & 4.310 & 3.994 \\
1996 & 4.216 & 3.663 \\
1997 & 3.670 & 3.264 \\
1998 & 4.758 & 4.258 \\
1999 & 4.372 & 4.108 \\
2000 & 4.855 & 2.874 \\
2001 & 4.297 & 1.172 \\
2002 & 16.231 & 1.574 \\
\hline Totales & 68.145 & 68.145 \\
\hline
\end{tabular}

Si a esos datos y estimaciones se añade el hecho de que, a pesar de los esfuerzos de investigación que se están realizando por parte de los especialistas biomédicos no se prevé que en el futuro próximo se descubra una cura o vacuna que en definitiva pongan coto a la enfermedad (AIDS Vaccine, 2003; Klausner et al., 2003; Mugyenyi, 2002; ONUSIDA/OMS, 2003), es imprescindible que en las instituciones del sector salud se asegure el diseño e instrumentación de programas de salud pública cuyos objetivos se orienten a: a) evitar nuevas infecciones por $\mathrm{VIH}$ en los diferentes sectores de la población, y b) mejorar la oportunidad y calidad de los servicios que se brindan a las personas que viven con el VIH o el SIDA en sus diferentes estadios, tanto en lo que corresponde a la atención de tipo médico como a la psicológica y social. Sobre el respecto, podemos adelantar que para conseguir ambos objetivos es fundamental que esos programas se afirmen en propuestas de corte multidisciplinario, en los cuales la psicología tiene sin lugar a dudas mucho que aportar (véase Arranz y Bayés, 1988; Ardila, 1995; Bayés, 1992).

En efecto, como acertadamente señala Bayés (1995a, 1995b), no hay que pasar por alto que tanto en los ámbitos de la prevención como en el de la intervención, propiamente dicha, el análisis y evaluación de la conducta y de los factores psicológicos que les subyacen, constituyen dos actividades que revisten de singular importancia. El problema, sin embargo, radica en asegurar que el análisis y evaluación de ambos aspectos sean realmente tenidos en cuenta al momento de diseñar e instrumentar los programas; de otra suerte, tal y como ha ocurrido en nuestro país, el discurso y la práctica institucionales sobre la materia no pasarán de estar fundados, sí, en muy buenas intenciones, aunque seguirán estando limitados en cuanto a la efectividad de las acciones y en la obtención 
de sus resultados (Piña, 2003; Piña, en prensa).

Para justificar lo antes dicho, en el siguiente apartado nos damos a la tarea de revisar algunos documentos y trabajos de investigación realizados en el contexto de las instituciones del sector salud en México, con la aclaración de que no pretendemos agotar aquí la discusión sobre la manera en la que se concibe y aborda el problema en cuestión, sino simplemente destacar diversos puntos que consideramos conveniente tener presentes, previo a la discusión que se expone en el apartado final de este escrito.

\section{BREVE REVISIÓN DEL DISCURSO Y LA INVESTIGACIÓN SOBRE VIH/SIDA EN LA SALUD PÚBLICA INSTITUCIONAL}

En la introducción del Programa de Acción: VIH/SIDA e Infecciones de Transmisión Sexual (ITS), presentado por la Secretaría de Salud en el ámbito nacional (Secretaría de Salud, 2002), se lee lo siguiente; citaremos en extenso:

En los programas de prevención y control del VIH/SIDA e ITS prevalece una visión médico-asistencial que no incorpora otros elementos fundamentales, como son: el trabajo en defensa de los derechos humanos, las acciones para disminuir el estigma y la discriminación, los planes con enfoque de género, grupos de auto ayuda, servicios de apoyo comunitario y asesoría legal... Se requiere, además, ampliar la respuesta y focalizar las acciones preventivas a las poblaciones más afectadas o con mayor vulnerabilidad: mujeres, jóvenes, Hombres que tienen Sexo con otros Hombres (HSH), Trabajadoras y Trabajadores del Sexo Comercial (TSC) y Usuarios de Drogas Inyectables (UDI), poblaciones móviles, personas privadas de la libertad e indígenas, así como a los contextos que aumentan el riesgo y la vulnerabilidad al VIH/SIDA e ITS (Secretaría de Salud, 2002; pp. 10).

Como se podrá notar, no obstante que hay un reconocimiento explícito sobre la visión médico-asistencial que todavía prevalece en la gran mayoría - si no es que en la totalidad- de los programas de prevención y control del VIH/SIDA, cuando se hace referencia a qué otros elementos habría que incorporar en tales programas, también explícita pero inadvertidamente se deja de lado uno que desde nuestra óptica es fundamental para alcanzar el objetivo de prevenir nuevas infecciones por VIH, a saber, el relacionado con los factores psicológicos que subyacen a las conductas de riesgo o de prevención.

Mencionar, por ejemplo, que «la complejidad del VIH/SIDA requiere estrategias integrales que incluyan factores $p s i$ cológicos ${ }^{3}$, sociales, culturales, religiosos, étnicos, sexuales y económicos» (Secretaría de Salud, 2002; pp. 20), puede, ciertamente, suponer muchas cosas, pero a la vez no decir nada. Valdría la pena preguntarnos, por lo tanto, a qué se refieren las autoridades del sector salud cuando hacen mención de los factores psicológicos. Desafortunadamente, ni a lo largo del citado documento ni en otros elaborados por dichas autoridades se encontró una definición o una simple idea acerca de lo que entienden por factores psicológicos, de cómo se presume se relacionan con la práctica de las conductas de riesgo o prevención, de cuál y cómo es que se le relacionan con factores biológicos y sociales, principalmente (p.ej., Izáosla et al., 1999; Secretaría de Salud, 1997).

Asimismo, asegurar que la prevención sexual de la infección por VIH/SIDA puede conseguirse una vez que se pongan en marcha acciones estratégicas, como por

\footnotetext{
${ }^{3}$ Cursivas de los autores.
} 
ejemplo, «incrementar la frecuencia y difusión de campañas que se apeguen a los lineamientos técnicos y que promuevan conductas sexuales exentas de riesgo...», o bien, "consolidar e incrementar programas de educación de conductas sexuales saludables para grupos vulnerables, grupos en riesgo y la población en general» (Secretaría de Salud, 2002; p. 37), puede para el lector resultar coherente, pero no por ello fácil de conseguir; abordaremos brevemente este punto.

En México, la práctica de la investigación sobre el problema del VIH/SIDA, especialmente la que se realiza con el propósito de derivar programas y acciones estratégicas como las apuntadas en el párrafo anterior, se ha justificado, en el plano institucional -entiéndase el sector salud-, en una orientación eminentemente epidemiológica y social. En esa dirección apuntan, por ejemplo, trabajos en los que se ha tratado de identificar los conocimientos, actitudes y prácticas de riesgo en diferentes sectores de la población, apoyándose para dicho efecto en las conocidas como encuestas CAP (Secretaría de Salud, 1989). También, hay otras que se han centrado en el uso de condón en hombres con parejas no estables (p.ej., Nieto e Izazola, 1999), o bien otras que se han propuesto identificar qué características demográficas o educativas influyen para que los adolescentes hablen sobre SIDA y con qué interlocutores (véase Gayet, Rosas, Magis y Uribe, 2002).

Son, en términos generales, trabajos en los que, no obstante se han obtenido algunos resultados interesantes, por sus propias características presentan el inconveniente de limitarse en su análisis a un recuento de frecuencias, porcentajes, comparaciones de medias y demás, de variables demográficas (como edad, sexo, nivel escolar, etcétera), psicologicas (conocimientos y actitudes, por ejemplo), conductas de riesgo (uso inconsistente de condón) $u$ otros tipos de conductas (hablar de SIDA con otro adolescente, el profesor o algún padre de familia). Sin embargo, qué es lo que nos dice que los estudiantes universitarios poseen un buen nivel de conocimientos ímedido a partir del porcentaje de respuestas correctas); adicionalmente, que reportan ciertas actitudes, por ejemplo hacia el condón y su uso; o bien, que practican tales o cuales conductas de riesgo, como tener relaciones sexuales sin protección, es decir, sin usar condón.

Dicho en otras palabras, suponer que a partir de la descripción aislada de algunas cuantas variables (conocimientos y actitudes, por ejemplo) será suficiente para entender por qué una persona se comporta de manera arriesgada o practica conductas de prevención, resulta a todas luces insuficiente. Es preciso aclarar, por lo demás, que no nos estamos refiriendo tan sólo a la forma en la que conceptos como el de conocimientos y el de actitudes son definidos y utilizados en esas investigaciones, sino sobre todo a la viabilidad y pertinencia de la orientación epidemiológica y social para dar cuenta de variables que presumiblemente forman parte del discurso sobre lo psicológico.

No hay que pasar por alto, y ello dicho por las propias autoridades del sector salud, que el programa global de VIH/SIDA e ITS fue de todos los programas institucionales -incluyendo los de prevención del cáncer, enfermedades cardiovasculares, diabetes mellitus, etc.el que más bajo desempeño tuvo en el año 2001, cumpliéndose con tan sólo $30 \%$ de las actividades resumidas en el indicador compuesto; si bien es cierto que se señala que para el siguiente año se observó un incremento porcentual de $55 \%$ en el cumplimiento de las actividades, el programa en cuestión ocupó el penúltimo lugar de todos los programas, mientras que el de diabetes mellitus ocupó el último. 
Si, como mencionan Hernández, Garrido y López (2000), el principal objetivo de la investigación epidemiológica tiene que ver con la necesidad de desarrollar un mejor conocimiento de la distribución de las enfermedades y eventos de salud en la población, así como contribuir al descubrimiento y caracterización de las leyes que regulan o influyen en estas condiciones, en el caso que nos ocupa todo parece indicar que ese tipo de investigación ha evidenciado múltiples limitaciones, sobre todo si su puesta en marcha no corre al parejo de otro tipo de investigación; nos referimos concretamente a la investigación sobre la conducta y los factores psicológicos que les subyacen. Como agudamente señala Bayés (1995a):

Lo que sí debería deducirse de lo anterior es -con vacuna o sin ella- la necesidad de poseer el mejor conocimiento posible de los factores susceptibles de facilitar o dificultar la práctica de los comportamientos de riesgo, con el fin de elaborar estrategias capaces de eliminarlos o cambiarlos por comportamientos de prevención eficaces. Y esto, como veremos enseguida, no es sólo un problema de campañas de información, supone investigación. Investigación científica seria, de mayor complejidad y dificultad sin duda, pero con el mismo grado de exigencia en evaluaciones estrictas independientes, con el mismo grado de «dureza'» que la investigación biomédica (Bayés, 1995a; pp. 3-4).

Constituye, por lo tanto, un tipo de investigación necesaria y que debiera concebírsele como complemento de la investigación epidemiológica y social. No hay que pasar por alto que la investigación sobre las conductas de riesgo/prevención y de los factores psicológicos que les subyacen representa el único medio a partir del cual será posible dar cuenta de cómo, por qué y bajo qué circunstancias los individuos optan por la práctica de uno u otro tipo de conducta, identificar qué factores psicológicos las hacen más o menos probables, y a partir de ahí, derivar programas y estrategias dirigidos a prevenir nuevas infecciones por VIH (Bayés, 1990; Piña y Bullé, 1994; Ribes, 1990).

\section{PSICOLOGÍA Y VIH/SIDA: INVESTIGACIÓN Y PREVENCIÓN}

En líneas atrás se ha señalado la importancia de realizar investigación que nos permita arribar a un mejor conocimiento de los factores que facilitan o dificultan la práctica de conductas de riesgo, y con base en los resultados, estar en una mejor posición de generar estrategias de intervención que se orienten hacia el cambio de dichas conductas por conductas de prevención.

Sin embargo, no sólo se trata de realizar investigación; debe, asimismo, tomarse en consideración el modelo conceptual que sobre lo psicológico se tenga en este camino que hay que transitar para el entendimiento de la conducta humana. En México, así como en otros países, se han desarrollado cantidades importantes de investigaciones que parten de modelos conceptuales que enfatizan el papel de determinadas variables de corte racional o cognoscitivo (conocimientos, actitudes, creencias, intención, percepción de riesgo, entre otras), dando por sentado que al incidir sobre tales variables será posible lograr cambios en la conducta humana. Tal y como se ha comentado en otro lugar (Robles y Moreno, 2002), los resultados de investigaciones con base en esos modelos han demostrado ser inconsistentes, y en consecuencia, no permiten entender y explicar por qué una persona se comporta de manera arriesgada a pesar de tener un buen nivel de conocimientos o ciertas actitudes favorables hacia las conductas de prevención, por ejemplo. 
Una propuesta alternativa a esos modelos la constituye el modelo psicológico de salud biológica propuesto por Ribes (1990). Desde esta perspectiva, hablar de conducta es hablar de la interacción del individuo con su ambiente; en la medida en la que la conducta supone un proceso interactivo entre el organismo y el ambiente, la conducta es interconducta (Ribes y López, 1985). En relación con el campo de los problemas de la salud, en el modelo psicológico - cuyos dos antecedentes fundamentales se encuentran en una teoría de la conducta (Ribes y López, op cit.) y una teoría de la personalidad (Ribes y Sánchez, 1990) -, la dimensión psicológica de la salud puede desagregarse en tres factores o componentes:

a) La forma en que la conducta participa en la modulación de los estados biológicos, referidos a los diferentes subsistemas biológicos de respuesta, casos del inmune, endocrino, nervioso central, etcétera;

b) Las competencias que definen la efectividad para interactuar con toda clase de situaciones que pueden afectar la salud, $y$

c) Las maneras consistentes de comportarse de un individuo.

Son tres factores que, como señala Ribes (1990), involucran una diversidad de funciones psicológicas, y su proceso será determinante de los resultados biológicos en términos de salud o enfermedad. En el caso que nos ocupa, el que una persona practique un tipo particular de conducta instrumental - e.g., tener relaciones sexuales con penetración y no utilizar condón-, determinará en menor o mayor medida la probabilidad de que resulte infectada por el VIH. Empero, en tanto que este tipo de conducta u otras más forman parte de la fase de resultados del modelo psicológico antes mencionado, es indispensable dar cuenta de qué y cómo es que diversos factores psicológicos son los que finalmente facilitan o impiden que esa $u$ otras conductas se practiquen. A continuación resumimos algunos estudios realizados en nuestro país, que se han interesado en investigar sobre este último aspecto.

Con el objeto de identificar qué factores psicológicos correlacionan o predicen algunas conductas instrumentales de riesgo/prevención asociadas con el VIH/SIDA, Piña y Corral (2001), con una muestra de 421 estudiantes universitarios, utilizando un análisis de regresión múltiple por pasos identificaron siete predictores significativos -e.g., edad, área de estudio, nivel escolar, creencias religiosas, conocimiento sobre el riesgo de promiscuidad, conocimiento sobre el uso del condón- sobre siete conductas instrumentales de riesgo -e.g., debut sexual a temprana edad, elevada frecuencia de relaciones sexuales, relaciones con múltiples parejas, baja frecuencia de uso de condón con parejas estables o múltiples parejas-; tales predictores en conjunto explican $25 \%$ de la varianza. Asimismo, mediante un análisis factorial confirmatorio se encontró que cinco motivos -e.g., para tener relaciones, para tener relaciones en los últimos seis meses, para no usar condón, para tener múltiples parejas y para tener relaciones con penetración por el ano- se constituyeron en predictores altamente significativos de las mismas conductas instrumentales de riesgo, explicando $88 \%$ de la varianza.

En otro trabajo (Piña y Werner, en prensa), que tuvo como objetivo identificar predictores psicológicos del uso de condón en una muestra de 359 mujeres, todas estudiantes de educación superior, mediante un análisis de regresión (método Weighted Estimation) se encontró que del total de variables consideradas, que incluían a los conocimientos, creencias y 
motivos ${ }^{4}$, únicamente estos últimos demostraron un elevado poder predictivo sobre las dos conductas instrumentales de riesgo: frecuencia de uso de condón en toda la vida $\left(R^{2}=0,5410\right.$, explicando $54,10 \%$ de la varianza) y uso de condón con parejas ocasionales $\left(R^{2}=\right.$ 0,5196 , explicando $51,96 \%$ de la varianza).

También, Piña, Urquidi, Molina y Cota (en revisión), en una investigación en la que participaron 317 estudiantes de dos instituciones de educación superior, con un análisis de regresión (método Weigthed Least Squares) se encontró que, de cuatro variables psicológicas, que incluŕan a los conocimientos, creencias, circunstancias sociales y motivos, las dos últimas predijeron cinco conductas de riesgo: debut sexual $\left(R^{2}=0,0790\right.$, explicando $7,90 \%$ de la varianza), número de parejas en toda la vida $\left(R^{2}=0,3477\right.$, explicando $34,77 \%$ de la varianza), uso de condón en toda la vida $\left(R^{2}=0,1701\right.$, explicando $17,01 \%$ de la varianza), relaciones con parejas ocasionales $\left(R^{2}=\right.$ 0,5296 , explicando $52,96 \%$ de la varianza) y uso de condón con parejas ocasionales $\left(R^{2}=0,3776\right.$, explicando $37,76 \%$ de la varianza).

Por su parte, Robles y Moreno (2000) llevaron a cabo un estudio con una muestra de 1559 estudiantes de educación superior, a quienes se les administró un cuestionario conformado por 92 reactivos de opción múltiple, y en el cual se evaluaron ocho prácticas de riesgo: relaciones anales activas y pasivas, relaciones orales activas y pasivas, relaciones con parejas ocasionales, relaciones homosexuales, relaciones en grupo y frecuencia de uso de condón, entre otras. Los resultados indican que $49,5 \%$ de los estu-

\footnotetext{
4 La definición de esos conceptos se realizó respetando en todo momento la lógica y los supuestos de una teorfa de la conducta (Ribes y López, 1985; Ribes y Sánchez, 1994) y una teoría de la personalidad (Ribes y Sánchez, 1990).
}

diantes reportaron tener experiencia sexual, la mitad de los cuales las habían iniciado entre los 16 y 18 años. Cerca de $38 \%$ mencionó tener entre una y cinco relaciones sexuales al mes, y $30 \%$ más de seis relaciones. Por otro lado, $85 \%$ señaló tener relaciones con una pareja estable, mientras que del restante $15 \%$, alrededor de $42 \%$ las tenía con parejas ocasionales, con profesionales del sexo o con otros tipos de pareja. Un dato especialmente importante es que únicamente $8,7 \%$ reportó usar el condón como medida preventiva y $12,9 \%$ cuando se desconocían los antecedentes sexuales de la pareja. Destaca, por último, el uso de condón en las diferentes prácticas: $46,7 \%$ lo usa siempre cuando se tienen relaciones anales activas, $10,3 \%$ cuando son orales activas, $43,5 \%$ en orales pasivas, $77 \%$ con parejas ocasionales y $\mathbf{3 0 , 8} \%$ cuando se tienen relaciones homosexuales.

Por otro lado, Robles y Moreno (en prensa), en un estudio realizado con 2 254 estudiantes de educación superior, se dieron a la tarea de evaluar conocimientos y conductas sexuales de riesgo/prevención. Destacan, entre otros, los siguientes resultados: un análisis de varianza detectó diferencias significativas en los conocimientos generales sobre VIH/SIDA entre los estudiantes de las diferentes carreras $(F(5 / 2248)=27,8872$; $p<0,001\}$, mientras que la prueba de Scheffé indicó que las diferencias se presentaron entre los estudiantes de las carreras de enfermería y odontología, quienes obtuvieron puntuaciones mayores, comparados con los estudiantes de administración, psicología, arquitectura y derecho. Se observó, también, que por área de conocimiento sobre VIH/SIDA, los valores más bajos se encontraron en las áreas de epidemiología $(47,05 \%)$ y etiología $(54,03 \%)$, mientras que los más altos se obtuvieron en las áreas de prevención $(83,09 \%)$, sintomatología $(82,13 \%)$ y transmisión $(78,07 \%)$. Al 
comparar el nivel de conocimientos entre los estudiantes con y sin experiencia sexual, se detectaron diferencias significativas en las áreas de etiología $\{F(1 / 2$ 169) $=13,6166 ; p=0,0002\}$, prevención $\{F(1 / 2169)=7,4708 ; p=0,006\}$ y en el porcentaje global de conocimientos $\{F(1 / 2169)=11,3364 ; p=0,0008\}$, lo que indica que los estudiantes que han tenido relaciones sexuales muestran un nivel más elevado de conocimientos que quienes no las han tenido.

Respecto de las conductas sexuales, del total de la muestra, $1117(49,5 \%)$ ya habían debutado sexualmente, con cerca de $55,1 \%$ haciéndolo entre los 16 y 18 años. $41,9 \%$ mencionó que tenían relaciones sexuales esporádicas y sólo $2,5 \%$ las realiza más de 20 veces al mes. Por otro lado, $87,7 \%$ señaló que tenía sus relaciones con una pareja estable, observándose que $50,8 \%$ de los estudiantes utilizaba condón de manera inconsistente, y el restante $49,2 \%$ lo utilizaba consistentemente. Al preguntársele a los estudiantes por qué utilizaban condón, $61,6 \%$ respondió que para evitar embarazos, $13,8 \%$ porque desconocía los antecedentes sexuales de la pareja, $10,3 \%$ porque consideraba que había riesgo de alguna infección de transmisión sexual, mientras que sólo $2,1 \%$ porque su pareja se lo había pedido.

Finalmente, Moreno, Robles, Frías y Rodríguez (2003), en una investigación que contó con una muestra de 524 estudiantes de educación superior sexualmente activos, se evaluaron diferentes conductas instrumentales de riesgo: frecuencia de relaciones sexuales, tipos de parejas con la que se tienen relaciones sexuales, uso y frecuencia de uso de condón, principalmente. De los resultados destacan los siguientes: $55 \%$ de los estudiantes reportó usar condón, aunque de éstos $52 \%$ lo hizo inconsistentemente, es decir, prácticamente la mitad de los estudiantes que mencionaron que lo utiliza- ban lo hacían en todas y cada una de sus relaciones sexuales con penetración. Otro dato interesante, es que de los estudiantes que mencionaron tener relaciones esporádicas, únicamente $47 \%$ reportó que utilizaba condón siempre, $32 \%$ la mayoría de las veces, $15 \%$ la mitad de las veces y $6 \%$ nunca. Un análisis con la Ji cuadrado reveló, para los estudiantes que reportaron tener relaciones sexuales con mayor frecuencia o relaciones sexuales tipificadas como "arriesgadas», diferencias estadísticamente significativas en lo tocante a la frecuencia de uso de condón $\chi^{2}(3,0,000 ; p<0,05)$, indicando que la frecuencia de relaciones sexuales con penetración es inversa a la frecuencia de uso de condón.

Sobre el tipo de pareja con la que se usa condón, $\mathbf{5 7} \%$ de los estudiantes mencionó que lo utiliza con su pareja estable, $16 \%$ cuando tienen relaciones con parejas eventuales u ocasionales, $14 \%$ con amistades y $9 \%$ con otro tipo de pareja; es interesante hacer notar que únicamente $4 \%$ de los estudiantes señaló que usaba condón cuando tenían relaciones con profesionales del sexo, mientras que $0,7 \%$ con personas desconocidas; estos datos sugieren que $43 \%$ de los estudiantes práctica conductas instrumentales de riesgo. Un análisis con la Ji cuadrado evidenció diferencias significativas para el tipo de pareja con la que se usa condón $\chi^{2}(5,0,000 ; p<0,05)$. Por último, para las razones o motivos que subyacen a la conducta uso de condón se encontró que $53 \%$ de los estudiantes reportó que lo usa para evitar embarazos, $19 \%$ cuando desconocía los antecedentes sexuales de sus parejas y $15 \%$ cuando consideraba existía riesgo de alguna infección sexual, principalmente; el análisis con la Ji cuadrado reveló diferencias significativas en este rubro, en donde los hombres evaluados señalan esas razones o motivos para usar condón $\chi^{2}(4,0,000 ; p<0,05)$, es decir, como medida anticonceptiva y no 
para los fines de evitar una infección sexual, como la del VIH.

¿Qué es lo que tienen en común estos trabajos y cuáles son las ventajas que presumiblemente poseen para los efectos de prevenir la infección por VIH, al menos en muestras de estudiantes universitarios? En primer lugar, es necesario destacar que, frente a otros modelos u orientaciones teóricas que han surgido desde la psicología, como por ejemplo el modelo de la acción razonada de Ajzen y Fishbein (1980) y Fishbein y Azjen (1975), el de reducción del riesgo (ARRM, por sus siglas en inglés) de Catania, Kegeles y Coates (1990) o el modelo de creencias de salud de Rosenstock (1974), entre otros, el modelo psicológico de salud biológica nos ofrece: a) un marco teórico y conceptual específico -el interconductual-; b) define con precisión su propio objeto de estudio -la conducta interactiva-; c) delimita también con precisión la dimensión psicológica en la salud - las variables o factores que son pertinentes-, pero sobre todo, d) tiene en cuenta que la conducta de los individuos no puede, salvo para propósitos exclusivamente analíticos, disociar los componentes «racionales" de otros relacionados con el lenguaje de los afectos, las emociones y los motivos, principalmente (véase Morales y Piña, 1995; Piña y Bullé-Goyri, 1994; Rodríguez, Moreno, Robles y Díaz-González, 2000).

En segundo lugar, que el modelo psicológico de salud biológica especifica el espectro de su aplicabilidad en el contexto profesional (Díaz-González y Carpìo, 1996; Rodríguez y Landa, 1996; Rodríguez y cols., 2000). Para el caso que nos ocupa, el de la prevención de la infección por VIH, el primer paso a dar se justifica en el quehacer de la investigación, de cuyo testimonio se ha dado cuenta con algunos ejemplos; en éstos, se ha subrayado el importante papel que una diversidad de variables juegan como predictores o variables que correlacionan con varios tipos de conductas instrumentales de riesgo. El siguiente paso consiste en traducir esos hallazgos en estrategias para el cambio de las conductas instrumentales de riesgo por conductas instrumentales de prevención. En este nivel, se trata de dotar a las personas de los recursos competenciales y las destrezas que les permitan enfrentar los requerimientos de su vida cotidiana, promover el establecimiento de estilos interactivos saludables y desarrollar las conductas instrumentales de prevención apropiadas, tales como: saber qué es el SIDA, cómo se transmite y qué medidas preventivas existen; evitar o posponer una relación sexual, y en caso de tenerla, utilizar condón correcta y consistentemente; tener relaciones sexuales con una sola pareja e investigar la historia sexual de su (s) pareja (s), básicamente (Rodríguez y cols., 2000), además de tener las habilidades competenciales para negociar con éxito el uso del condón (Robles et al., 2003).

En esta dirección, recientemente, DíazGonzález, Rodríguez, Moreno y Frías (2003) propusieron, con base en este modelo psicológico de salud biológica, una serie de lineamientos generales para el diseño de un instrumento de evaluación de las competencias funcionales relacionadas con el VIH/SIDA, tomando como herramienta metodológica el análisis contingencial. En la propuesta del instrumento se presentan ítemes que permiten evaluar aspectos en donde lo importante es la forma de relacionarse sexualmente, los tipos de situaciones que dan contexto a las relaciones sexuales, el papel que juegan otras personas significativas a partir de su propia conducta y los efectos producidos por las conductas sexuales de riesgo o de prevención. Para cada una de estas categorías microcontingenciales se presentan opciones de respuesta que evalúan los cuatro niveles 
funcionales en los que pueden tener lugar las competencias efectivas en una situación interactiva: no instrumental, instrumental, extrasituacional y transituacional (Ribes, 1990). En este tipo de investigación, que actualmente se encuentra en su fase piloto, se está trabajando con tres tipos poblaciones: personas que viven con VIH, estudiantes de nivel medio y estudiantes de educación superior.

En resumen, si hoy en día sabemos que la única forma eficaz de evitar nuevas infecciones por VIH es mediante la modificación de las conductas instrumentales de riesgo por conductas instrumentales de prevención, es imperativo que desde un punto de vista psicológico se realicen esfuerzos de investigación tendientes a conseguir: a) un mejor y un más completo entendimiento de qué factores psicológicos son las que facilitan o impiden que tales conductas se practiquen, y b) un diseño y puesta en marcha de programas y estrategias dirigidos a la modificación de la conducta, susceptibles de ser evaluadas sistemáticamente y probar así su eficacia real.

Prevenir es predecir (Fernández y Mercado, 1987), y predecir, en términos psicológicos, es reconocer la probabilidad de que un individuo se comportará de una manera u otra, es decir, que practicará una conducta de riesgo - tener relaciones sexuales sin protección- o una de prevención - se abstendrá de tener una relación sexual o, en caso de tenerla, utilizará condón- (Piña y Obregón, 2003). De ello deriva, entonces, que para prevenir nuevas infecciones por VIH debemos ser capaces de identificar qué y cómo operan determinados factores psicológicos -estilos interactivos, competencias, motivos, etcétera- en relación con cualesquiera conductas instrumentales de riesgo o de prevención.

Como acertadamente propone Ardila (2000), el psicólogo de la salud que tra- baja en el ámbito de la prevención primaria requiere un sólido entrenamiento en metodología científica, un manejo adecuado de las políticas de salud y una indiscutible capacidad para trabajar en equipos multidisciplinarios. Pero, también, consideramos que es fundamental contar con un marco teórico de referencia que le permita sugerir hipótesis de investigación y a la vez como guía para coadyuvar en el diseño e instrumentación de programas y estrategias encaminados a lograr una real y efectiva prevención de nuevas infecciones por VIH.

Finalmente, a partir de un modelo psicológico de salud biológica se ha dado cuenta de algunas investigaciones realizadas en los últimos tres años en nuestro país. Los resultados aquí reseñados nos permiten no sólo reconocer la pertinencia teórica del modelo, sino también su utilidad práctica en el quehacer de la investigación. La investigación empírica generada desde esta perspectiva, nos parece puede influir de manera decisiva en el diseño e instrumentación de programas y estrategias de prevención, orientados al cambio de conductas de riesgo por conductas de prevención. Esto último resulta especialmente importante, dado que incidir únicamente sobre el nivel de conocimientos o las actitudes que refieren los diferentes sectores de la población no ha mostrado hasta el momento su eficacia para promover el cambio en esas conductas, especialmente cuando se parte del supuesto de que, por ejemplo, conocer o saber algo, como conducta, se encuentra disociado del saber cómo, pues saber algo sólo puede tener sentido a partir de saber cómo (Ribes, 2002).

En este sentido, se debe procurar incorporar diversos factores que son claves en el modelo psicológico aquí comentado, sobre todo dos de ellos: los motivos y las competencias funcionales. La siguiente tarea consiste en diseñar y 
poner en marcha intervenciones preventivas que reconozcan las aportaciones hechas tanto por los especialistas en epidemiología como por otros pertenecientes a las disciplinas sociales, como parte de un esfuerzo colectivo y multidisciplinario que exige todo problema de salud, incluyendo el del VIH/SIDA (véase Tobón y Vinaccia, 2003).

\section{REFERENCIAS BIBLIOGRÁFICAS}

AIDS Vaccine (2003). International Annual Conference. New York. En Internet: httl://www.aidsvaccine2003.org.

Ajzen, 1., y Fishbein, M. (1980). Understanding attitudes and predicting social behavior. Englewood Cliffs, NJ: Prentice-Hall.

Anaya, L. (1997). Subestimación de la incidencia de SIDA en México, debida al retraso en la notificación. Revista SIDA-ETS, 3, 35-58.

Ardila, R. (1995). La respuesta de Colombia ante el SIDA: aportes psicológicos. En J.A. Piña (Comp.), SIDA: perspectiva psicológica de un problema de salud mundial (pp. 17-25). Hermosillo, México: Editorial Universidad de Sonora.

Ardila, R. (2000). Prevención primaria en psicología de la salud. En L.A. Oblitas y E. Becoña (Eds.), Psicología de la Salud (pp. 53-65). México: Plaza y Valdés Editores.

Arranz, P., y Bayés, R. (1988). SIDA y prevención: un enfoque multidisciplinario. JANO, Medicina y Humanidades, 34, 1326-1335.

Bayés, R. (1990). Aspectos psicológicos relacionados con el problema de la prevención del SIDA. En R. Nájera (Ed.), SIDA: de la biomedicina a la sociedad (pp. 377-390). Madrid: Ediciones de la Universidad Complutense.

Bayés, R. (1992). Aportaciones del análisis funcional de la conducta al problema del SIDA. Revista Latinoamericana de Psicología, 24, 35-56.

Bayés, R. (1995a). Psicología y SIDA. En J.A. Piña (Comp.), SIDA: perspectiva psicologica de un problema de salud mundial (pp. 1-15). Hermosillo, México: Editorial Universidad de Sonora.
Bayés, R. (1995b). SIDA y psicología. Barcelona: Martínez Roca.

Catania, J.A., Kegeles, S.M., y Coates, T.J. (1990). Towards an understanding of risk behaviors: An AIDS risk reduction model (ARRM). Health Education Quarterly, 17, 53-72.

CENSIDA (2003). Informe de casos de SIDA en México, hasta diciembre de 2002. México: Autor. En Internet: http://www.ssa.gob/epidemiología.

Díaz-González, E., y Carpio, C. (1996). Criterios para la aplicación del conocimiento psicológico. En J.J. Sánchez-Sosa, C. Carpio y E. Díaz-González (Comps.), Aplicaciones del conocimiento psicológico (pp. 39-49). México: UNAM/Sociedad Mexicana de Psicología.

Díaz-González, E., Rodríguez, M.L., Robles, S., Moreno, D., y Frías, B. (2003). El análisis contingencial como sistema analítico para la evaluación de competencias funcionales relacionadas con la prevención del VIH/SIDA. Psicología y Salud, 13, 149159.

Fishbein, M., y Ajzen, I. (1975). Belief, attitude, intention, and behavior: An introduction to theory and research. Reading, MA: Addison-Wesley.

Gayet, C., Rosas, C.A., Magis, C., y Uribe, P. (2002). Con quién hablan los adolescentes mexicanos sobre el SIDA. Salud Pública de México, 44, 122-128.

Izazola, J.A., Astaorla, L., Beloqui, J., Bronfman, M., Chequer, P., y Zacarías, F. (1999). Avances en la comprensión del VIH/SIDA: una visión multidisciplinaria. En J.A. Izazola (Ed.), EI SIDA en América Latina y el Caribe: una visión multidisciplinaria (pp. 21-44). México: Fundación Mexicana para la Salud/SIDALAC/ONUSIDA.

Izazola, J.A., Valdéz, M., Sánchez, H.J., y del Río, C. (1995). Mortalidad por SIDA en México de 1985 a 1992 . Tendencias y años perdidos de vida potencial. Salud Pública de México, 37, 140-148.

Klausnerm R., Fauci, A., Corey, L., Nabel, G., Gayle, H., Berkley, S., et al. (2003). Enhanced: The need for a global HIV vaccine enterprise. AIDS Science. Prevention and vaccine research. En Internet: http://www.aidscience.com/searcharticles.asp?/svalue=vaccines. 
Magis, C., y del Río, C. (2000). Epidemiología del VIH y del SIDA en México. En S. Ponce de León y S. Rangel (Eds.), SIDA. Aspectos clínicos y terapéuticos (pp. 1-10). México: McGraw-Hill Interamericana.

Morales, F. y Piña, J.A. (1995). La psicología y la salud hacia el siglo XXI: reflexiones desde la disciplina y la profesión. Psicología y Salud, 6, 127-138.

Moreno, D., Robles, S., Frías, B., y Rodríguez, M. (en prensa). Un estudio exploratorio de las variables de riesgo asociadas al uso de condón en una muestra de varones universitarios. Revista Iberoamericana de Psicología, 1.

Nieto, B., e Izazola, J.A. (1999). Uso del condón en hombres con parejas no estables de la Ciudad de México. Salud Pública de México, 41, 85-94.

ONUSIDA/OMS (2003). Resumen mundial de la epidemia de VIH/SIDA. En Internet: http://www.unaids.org.

Piña, J.A. (2003). Intervención psicológica en el área de la infección por el VIH: obstáculos y retos para los psicólogos de la salud. Terapia Psicológica, 21, 23-27.

Piña, J.A. (en prensa). Psicología y VIH/SIDA: aportaciones y asignaturas pendientes en México. Higiene (número especial: Nuevos enfoques en la Salud Pública en México).

Piña, J.A., y Bullé-Goyri, R. (1994). La psicología en el ámbito de la salud pública: perspectivas de un modelo psicológico para la investigación y la prevención. Psicología y Salud, 4, 177-185.

Piña, J.A., y Corral, V. (2001). Conocimientos y motivos asociados a comportamientos de riesgo y prevención relacionados con el SIDA. Avances en Psicología Clínica Latinoamericana, 19, 7-24.

Piña, J.A., y Obregón, F.J. (2003). Algunas reflexiones sobre el concepto de prevención en el contexto de la relación psicología y salud. En J.A. Piña, F.J. Obregón y J.A. Vera (Eds.), Psicología y Salud en Iberoamérica (pp. 53-72). Hermosillo, México: Secretaría de Salud/Universidad de Sonora/Centro de Investigación en Alimentación y Desarrollo, A.C.

Piña, J.A., y Werner, R. (en prensa). Predictores psicológicos del uso inconsistente de condón en mujeres. Cadernos Vida e Sáude (Brasil).
Piña, J.A., Urquidi, L.E., Molina, C., y Cota, M. (en revisión). Psychological predictors of risk behaviors related to HIV/AIDS in university students. AIDS and Behavior.

Ribes, E. (2002). El problema del aprendizaje: un análisis conceptual e histórico. En E. Ribes (Ed.), Psicología del aprendizaje (pp. 1-14). México: El Manual Moderno/U de G/UNAM.

Ribes, E., y López, F. (1985). Teoría de la conducta: un análisis de campo y paramétrico. México: Editorial Trillas.

Ribes, E., y Sánchez, S. (1990). El problema de las diferencias individuales: un análisis conceptual de la personalidad. En E. Ribes (Ed.), Psicología general (pp. 231-253). México: Editorial Trillas.

Ribes, E., y Sánchez, U. (1994). Conducta, juegos de lenguaje y criterios de validación del conocimiento. Acta Comportamentalia, 2, 57-86.

Robles, S., y Moreno, D. (2000). Evaluación de prácticas sexuales de riesgo en estudiantes universitarios. En La Psicología Social en México, Vol. VIII (pp. 748-755). Asociación Mexicana de Psicología Social.

Robles, S., y Moreno, D. (en prensa). Conocimientos y comportamiento sexual relacionados con la transmisión del VIH/SIDA en hombres y mujeres universitarios. En R. Hernández y A. Mata (Eds.), Comportamiento saludable: investigaciones. México: Asociación Mexicana de Comportamiento y Salud.

Robles, S., Moreno, D., Frías, B., Barroso, V., Rodríguez, M., Díaz-González, E., y Rodríguez, M.L. (2003, marzo). Effects of the training in assertive sexual communication on the risk sexual behavior in adolescentes. Trabajo presentado en el XVI Congreso Mundial de Sexología. La Habana, Cuba.

Rodríguez, M.L., y Landa, P. (1996). El análisis contingencial: una nueva alternativa para la psicología aplicable. En J.J. Sánchez-Sosa, C. Carpio y E. Díaz-González (Comps.), Aplicaciones del conocimiento psicológico (pp. 51-67) . México: UNAM/ Sociedad Mexicana de Psicología.

Rodríguez, M.L., Moreno, D., Robles, S., y Díaz-González, E. (2000). El SIDA desde el 
modelo psicológico de salud biológica. Psicología y Salud, 10, 161-175.

Rosenstock, I.M. (1974). Historical origins of the health belief model. Health Education Quarterly, 2, 324-473.

Secretaría de Salud (1989). Informe técnico. Evaluación del impacto de la estrategia educativa para la prevención del SIDA en México 1987-1988: estudiantes universitarios. México: Autor.

Secretaría de Salud (1997). Programa de fortalecimiento para la prevención y control del VIH/SIDA y otras enfermedades de transmisión sexual 1997-2000. México: CONASIDA.

Tobón, S., y Vinaccia, S. (2003). Modelo de intervención psicológica en el VIH/SIDA. Psicología y Salud, 13, 161-174.

Uribe, P., y Magis, C. (Eds.)(2000). La respuesta mexicana al SIDA: mejores prácticas. México: CENSIDA.

Valdespino, J.L., García, M.L., del Río, A., Loo, E., Magis, C., y Salcedo, E. (1995). Epidemiología del SIDA/VIH en México desde 1983 a marzo de 1985. Salud Pública de México, 37, 566-571. 